\title{
XMM-Newton observation of the ultraluminous quasar SDSS J010013.02+280225.8 at redshift 6.326
}

\author{
Yanli Ai, ${ }^{1 \star}$ A. C. Fabian, ${ }^{2}$ Xiaohui Fan, ${ }^{3 \star}$ S. A. Walker, ${ }^{2,4}$ G. Ghisellini, ${ }^{5}$ T. Sbarrato, ${ }^{6}$ \\ Liming Dou, ${ }^{7}$ Feige Wang, ${ }^{8}$ Xue-Bing $\mathrm{Wu}^{8,9}$ and Longlong Feng ${ }^{1}$ \\ ${ }^{1}$ School of Physics and Astronomy, Sun Yat-Sen University, Guangzhou 510275, China \\ ${ }^{2}$ Institute of Astronomy, Madingley Road, Cambridge CB3 OHA \\ ${ }^{3}$ Steward Observatory, University of Arizona, 933 North Cherry Avenue, Tucson, AZ 85721, USA \\ ${ }^{4}$ Astrophysics Science Division, X-ray Astrophysics Laboratory, Code 662, NASA Goddard Space Flight Center, Greenbelt, MD 20771, USA \\ ${ }^{5}$ INAF - Osservatorio Astronomico di Brera, via E. Bianchi 46, I-23807 Merate, Italy \\ ${ }^{6}$ Dipartimento di Fisica 'G. Occhialini', Università di Milano - Bicocca, Piazza della Scienza 3, I-20126 Milano, Italy \\ ${ }^{7}$ Center for Astrophysics, Guangzhou University, Guangzhou 510006, China \\ ${ }^{8}$ Department of Astronomy, School of Physics, Peking University, Beijing 100871, China \\ ${ }^{9}$ Kavli Institute for Astronomy and Astrophysics, Peking University, Beijing 100871, China
}

Accepted 2017 May 17. Received 2017 May 12; in original form 2017 February 26

\begin{abstract}
A brief Chandra observation of the ultraluminous quasar SDSS J010013.02+280225.8 at redshift 6.326 showed it to be a relatively bright, soft X-ray source with a count rate of about 1 count $\mathrm{ks}^{-1}$. In this article, we present results for the quasar from a 65-ks XMM-Newton observation, which constrains its spectral shape well. The quasar is clearly detected with a total of $\sim 460$ net counts in the $0.2-10 \mathrm{keV}$ band. The spectrum is characterized by a simple power-law model with a photon index of $\Gamma=2.30_{-0.10}^{+0.10}$ and the intrinsic $2-10 \mathrm{keV}$ luminosity is $3.14 \times 10^{45} \mathrm{erg} \mathrm{s}^{-1}$. The $1 \sigma$ upper limit to any intrinsic absorption column density is $N_{\mathrm{H}}$ $=6.07 \times 10^{22} \mathrm{~cm}^{-2}$. No significant iron emission lines were detected. We derive an X-rayto-optical flux ratio $\alpha_{\mathrm{ox}}$ of $-1.74 \pm 0.01$, consistent with the values found in other quasars of comparable ultraviolet luminosity. We did not detect significant flux variations either in the XMM-Newton exposure or between XMM-Newton and Chandra observations, which are separated by $\sim 8$ months. The X-ray observation enables the bolometric luminosity to be calculated after modelling the spectral energy distribution: the accretion rate is found to be sub-Eddington.
\end{abstract}

Key words: galaxies: active-galaxies: high-redshift-quasars: individual: SDSS J010013.02+280225.8.

\section{INTRODUCTION}

SDSS J010013.02+280225.8 (hereafter J0100+2802) is an ultraluminous quasar at a redshift of 6.326, which has an optical and infrared luminosity several times greater than any other high-redshift quasar and is inferred to host a $10^{10}-\mathrm{M}_{\odot}$ black hole (Wu et al. 2015). The quasar is clearly detected in an exploratory Chandra observation with an exposure of $14.8 \mathrm{ks}$ and is found to have a steep spectrum with $\Gamma=3.03_{-0.70}^{+0.78}$ derived from the 14 counts detected (Ai et al. 2016). This supermassive black hole might be growing with rapid accretion, as the bolometric luminosity yielded from X-ray to near-infrared observations was close to the Eddington luminosity (Wu et al. 2015; Ai et al. 2016). Considering the peculiar properties of all quasars discovered at $z \gtrsim 5$, which are powerful probes of cosmic reionization (Fan, Carilli \& Keating 2006), J0100+2802 sets

^E-mail: aiyanli@mail.sysu.edu.cn (YA); fan@as.arizona.edu (XF) the tightest constraints on models for massive black hole growth and evolution at early epochs (e.g. Shankar, Weinberg \& MiraldaEscudé 2009; Volonteri 2010).

In the Chandra observation, the X-ray-to-optical flux ratio of $\mathrm{J} 0100+2802$ is at the upper envelope of the observed $\alpha_{\text {ox }}$ values at a comparable ultraviolet luminosity, as reported in the Erratum (Ai et al. 2017) to the paper of Ai et al. (2017). Quasars are known to be variable and it is quite possible that this one has been caught in a bright state. The $z=7.1$ quasar, ULASJ1120+0641, is claimed to decrease in brightness by a factor of 4 between Chandra and XMM-Newton observations (Page et al. 2014), although debate exists (Moretti et al. 2014). There are hints of variation of J0100+2802 during the Chandra exposure, which is quite puzzling if no significant beaming effect has evolved. For high-redshift quasars, extended $\mathrm{X}$-ray lobes may be produced via Comptonization of the cosmic microwave background $(\mathrm{CMB})$ if relativistic electrons exist (Fabian et al. 2014). 
We proposed an XMM-Newton Director's Discretionary Time (DDT) observation of $\mathrm{J} 0100+2802$, which would yield an improved spectrum with greatly reduced errors in the spectral index and enable a search for any spectral features. Comparison of the flux with that from Chandra would provide a check on variability. Extended lobes produced from inverse Compton scattering of the CMB, which may extend over arcmin scales, could be detected with XMM-Newton. In this article, we report the spectral properties of this ultraluminous quasar from the XMM-Newton observation. Throughout this article, we adopt the $\Lambda \mathrm{CDM}$ cosmology parameters from the Planck Collaboration XVI (2014): $\Omega_{\mathrm{M}}=0.315, \Omega_{\Lambda}=0.685$ and $H_{0}=$ $67.3 \mathrm{~km} \mathrm{~s}^{-1}$. We define the power-law photon index $\Gamma$ such that $N(E) \propto E^{-\Gamma}$. For the Galactic absorption of SDSS J0100+2802, which is included in the model fitting, we use the value $N_{\mathrm{H}}=5.82$ $\times 10^{20} \mathrm{~cm}^{-2}$ (Kalberla et al. 2005). All uncertainties are given at $1 \sigma$, unless otherwise specified.

\section{XMM-NEWTON OBSERVATION AND DATA REDUCTION}

J0100+2802 was observed with XMM-Newton on 2016 June 29 for $65 \mathrm{ks}$ of Director's Discretionary Time. The European Photon Imaging Camera (EPIC) was operated in full-frame mode, with thin filters. The data were processed using the Science Analysis System (SAS) version 15.0.0. The time intervals of high-flaring background contamination were identified and excluded by inspection of the light curves in the 10-12 keV energy range. The total cleaned exposure times are 50 and $60 \mathrm{ks}$ for the PN and MOS cameras, respectively. Event patterns 0-12 were included in the MOS cameras, while for the PN camera we used patterns $0-4$. We constructed the images in four bands, $0.2-0.5,0.5-4,4-7$ and 7-10 keV, and then applied source detection simultaneously using the standard SAs task EDETECT_CHAIN.

We extract a spectrum of $\mathrm{J} 0100+2802$ from a 16 -arcsec radius region around the target in each EPIC detector, the source-extraction region corresponding to 60-70 per cent of the encircled energy fraction. The background was extracted from an adjacent source-free region with a larger radius. The spectra of the target from PN/MOS cameras were combined to form a single spectrum, with corresponding background spectra and response matrices also combined to form a single background spectrum and response matrix, using sAs task epicspeccombine. The EPIC spectra are then grouped in such a way that there are at least 25 counts in each energy bin. We only focus on spectrum analysis in this article.

\section{RESULTS}

As shown in Fig. 1, J0100+2802 is clearly detected in the XMMNewton EPIC images. The most accurate source position, from the Very Long Baseline Array (VLBA) 1.5-GHz image, lies within the astrometric uncertainties of both the optical Sloan Digital Sky Survey and the Chandra X-ray observation (Wang et al. 2017). The XMM X-ray position of the quasar given by SAS task EDETECT_CHAIN is $\sim 1.7$ arcsec away from the radio position, with a $1 \sigma$ position uncertainty of 0.6 arcsec.

The detected net count of $\mathrm{J} 0100+2802$ in the $0.2-10 \mathrm{keV}$ band is 460 . J0100+2802 is relatively soft, with weak detection in the hard $\mathrm{X}$-ray band, 2-10 keV (Fig. 1). It is detected individually in the $0.2-0.5$ and $0.5-4 \mathrm{keV}$ bands with false probability less than $10^{-10}$, while in the 4-7 keV band the detection significance of the quasar is close to $3 \sigma$ with a false probability of 0.015 . It is not detected in the $7-10 \mathrm{keV}$ band.
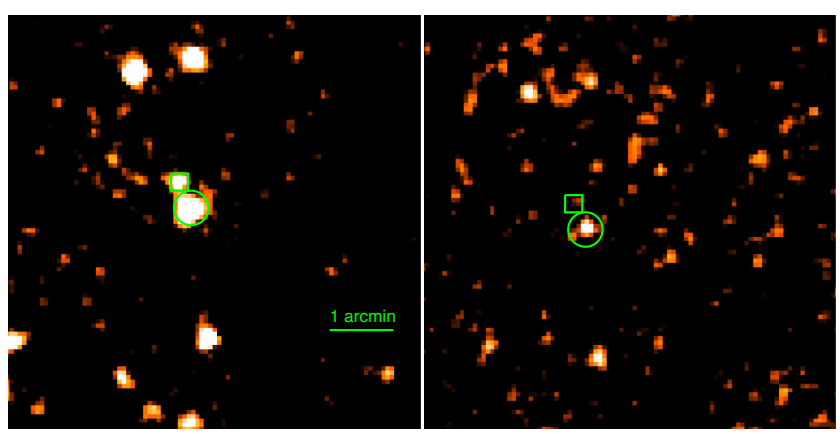

Figure 1. The 2-arcsec kernel smoothed XMM-Newton PN image of the $\mathrm{J} 0100+2802$ region of the sky in the observed $0.3-2 \mathrm{keV}$ (left panel) and $2-10 \mathrm{keV}$ (right panel) bands. The circle indicates the radius used to extract the spectrum and the square indicates the location of the nearby X-ray source SDSS J010013.95+280250.6. [A colour version of this figure is available in the online version.]

A nearby X-ray source, SDSS J010013.95+280250.6, which is detected in the Chandra observation 28 arcsec to the north-east of $\mathrm{J} 0100+2802$, is also detected in the XMM-Newton EPIC image (Fig. 1). This object is relatively faint in X-ray emission, with detected net counts of 80 in the $0.2-10 \mathrm{keV}$ band within a 15 -arcsec radius aperture in the EPIC images. It is not detected in the hard $\mathrm{X}$-ray band (2-10 keV in the observed frame), with an upper limit of $10^{-4}$ count $\mathrm{s}^{-1}$ estimated from the sensitivity maps using the SAS task esensmap for a logarithmic likelihood of 12. According to the point-spread function, the counts from this faint object, which falls in the source extraction region of our target quasar, are $\sim 8$ counts. Our target quasar therefore has little contamination from its emission.

The image of J0100+2802 appears slightly lopsided to the southeast, as shown in Fig. 2. The excess flux in the $0.5-2 \mathrm{keV}$ band is about $2 \times 10^{-16} \mathrm{erg} \mathrm{s}^{-1} \mathrm{~cm}^{-2}$. A deep Chandra image is required to distinguish several unresolved faint point sources from possible diffuse inverse Compton emission. If the latter occurs due to a jet from the quasar, then it may be detectable in the radio band below the mJy level.

We fitted the spectrum of J0100+2802 using XSPEC (v12.9; Arnaud 1996), using a simple power-law model modified by Galactic absorption. The fitted photon index is $\Gamma=2.30_{-0.10}^{+0.10}$. The fit is acceptable, with $\chi^{2}=24.7$ for 23 degrees of freedom (Fig. 3). We also fold the model with intrinsic absorption (at $z=6.326$ ). There is no significant improvement with $\Delta \chi^{2}$ of 1.7 and the $1 \sigma$ upper

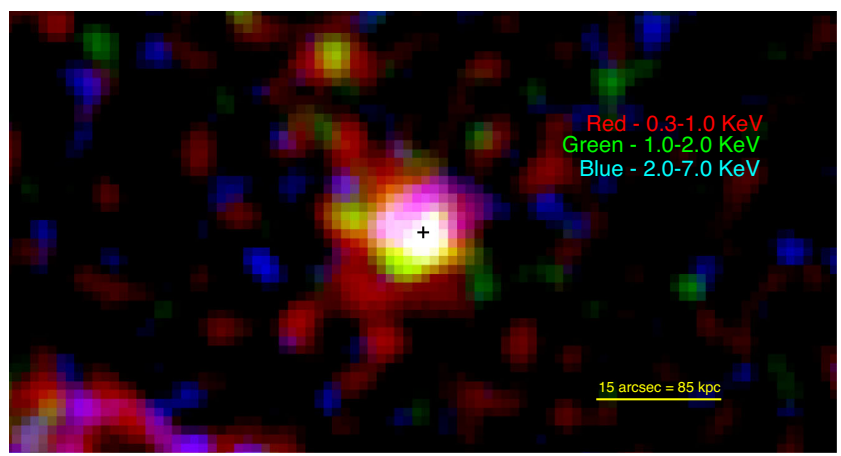

Figure 2. A RGB colour image of $\mathrm{J} 0100+2802$ using different bands from the EPIC PN image. Red shows soft X-ray emission (0.3-1.0 keV), green shows intermediate emission (1.0-2.0 keV) and blue shows hard X-ray emission. [A colour version of this figure is available in the online version.] 


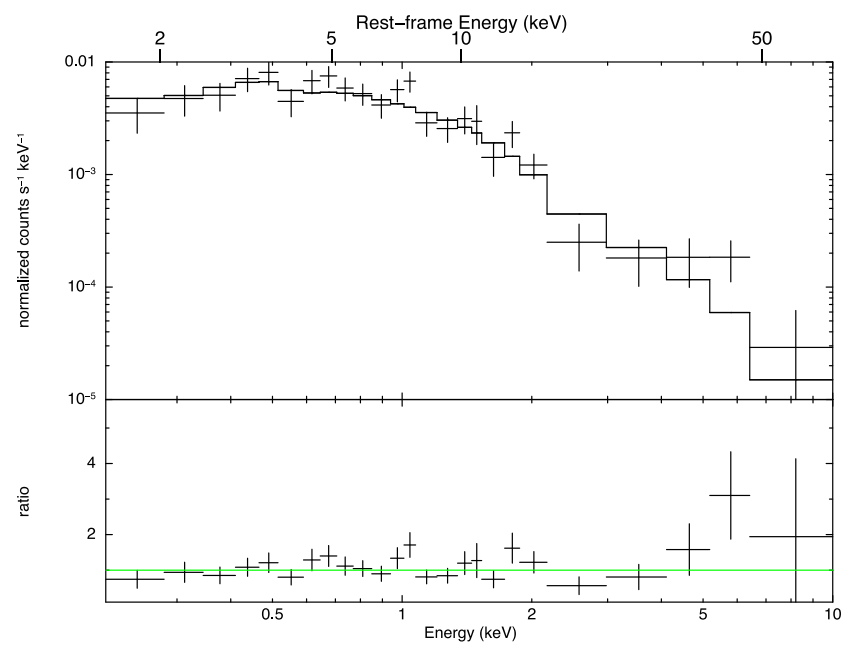

Figure 3. Upper panel: XMM-Newton spectrum of J0100+2802 and power-law model with fixed Galactic absorption. Lower panel: ratio of data to model. The relatively larger data-to-model ratio at energies greater than $4 \mathrm{keV}$ may be due to the dominance of X-ray background emission above $4 \mathrm{keV}$ for this quasar. [A colour version of this figure is available in the online version.]

limit of the intrinsic absorption column density is $N_{\mathrm{H}}=6.07 \times$ $10^{22} \mathrm{~cm}^{-2}$. There are residuals at energies in the range $5-10 \mathrm{keV}$, as shown in Fig. 3, possibly due to contamination from the background, as the source detection significance in this energy range is below $3 \sigma$. The rest-frame $2-10 \mathrm{keV}$ luminosity implied by the fit is $3.14_{-0.48}^{+0.53} \times 10^{45} \mathrm{erg} \mathrm{s}^{-1}$.

No Fe K emission-line feature appears to be present in the residuals and the $1 \sigma$ upper limit for the iron $\mathrm{K} \alpha$ equivalent width is $0.02 \mathrm{keV}$ (rest frame). There is a relatively larger data-to-model ratio at energies greater than $5 \mathrm{keV}$ (rest-frame $\sim 36 \mathrm{keV}$ ), possibly due to contamination from the statistical Poisson fluctuation of the background emission. As shown above, the detected significance of $\mathrm{J} 0100+2802$ at $4-7 \mathrm{keV}$ is only at the level of $3 \sigma$. Further deep exposures can help to justify whether the spectral shape of this quasar deviates from a simple power law at high energies.

The light curve for $\mathrm{J} 0100+2802$ is extracted and no significant variation is detected during the XMM-Newton exposure. We then compare the X-ray spectrum and flux between the Chandra and $X M M-N e w t o n$ observations with a time interval of about 8 months. First, the value of the inferred photon index from the XMM-Newton observation is within the errors of that from the Chandra observation, which is $\Gamma=3.03_{-0.70}^{+0.78}$. In other words, no statistical spectral shape variation was detected between the two observations for $\mathrm{J} 0100+2802$. Secondly, there is no detection of flux variation between the two observations, with the rest-frame 2-10 keV luminosity implied by the fit in the XMM-Newton observation consistent within errors with the value of $9.0_{-4.5}^{+9.1} \times 10^{45} \mathrm{erg} \mathrm{s}^{-1}$ from the Chandra observation. Finally, the residual at $\sim 1.2 \mathrm{keV}$, hinted at in the Chandra spectrum of $\mathrm{J} 0100+2802$, was not detected. The non-detection in the XMM-Newton observation indicates that the feature in the Chandra spectrum was probably due to instrumental lines (Bartalucci et al. 2014), although Poisson fluctuation cannot be excluded.

\section{DISCUSSION}

$\mathrm{J} 0100+2802$ is detected significantly in the XMM-Newton observation, with a total net count of 460 in the $0.2-10 \mathrm{keV}$ band. A simple power-law model provides acceptable fits to the spectrum with inferred photon index of $\Gamma=2.30_{-0.10}^{+0.10}$. The value of $\Gamma$ is consistent with the one found by Nanni et al. (2017). The $1 \sigma$ upper limit on any intrinsic absorption column density is $N_{\mathrm{H}}=6.07 \times 10^{22} \mathrm{~cm}^{-2}$. No significant iron emission lines were detected. With the X-ray spectral shape and luminosity well-constrained, we now discuss emission from the accretion disc with a broad-band energy spectral analysis for J0100+2802 and compare the spectral energy distribution (SED) of this quasar with those of other high- and low-redshift quasars.

\subsection{Black hole mass and disc luminosity of J0100+2802}

The black hole mass estimated by Wu et al. (2015) is $M_{\mathrm{BH}}=1.2 \times$ $10^{10} \mathrm{M}_{\odot}$. This is based on the virial method and is therefore affected by an uncertainty of factor 3 (acknowledged by Wu et al. 2015). The bolometric luminosity, assumed isotropic, given by Wu et al. (2015) is $L_{\text {bol }}=1.6 \times 10^{48} \mathrm{erg} \mathrm{s}^{-1}$ and includes the infrared and X-ray emission (following Shen et al. 2011). The corresponding opticalUV emission is nearly half of that (Calderone et al. 2013). The other half is reprocessed emission in the infrared by the absorbing torus surrounding the disc, plus the $\mathrm{X}$-ray emission produced by the corona sandwiching the disc. The latter could indeed be energized by the gravitational energy of the accreting matter.

Both the black hole mass and the accretion luminosity are huge and motivate us to explore alternative methods to measure them reliably. A proper accretion luminosity estimate should exclude the infrared reprocessed emission and take into account possible anisotropies. We therefore use a standard Shakura \& Sunyaev (1973) accretion-disc model to fit the observed optical-UV flux, while the corona X-ray emission is treated phenomenologically by adding a power law starting below the peak of the disc emission, ending with an exponential cut. This component requires three parameters: normalization, slope and cut frequency.

We are aware of the limitations connected with the use of the Shakura-Sunyaev disc model, due mainly to the following: (i) the spin is assumed to be zero; (ii) all relativistic effects are neglected and (iii) the disc is assumed to be geometrically thin and optically thick. The first assumption would lead to a lower limit on the black hole mass and an upper limit on the accretion rate, as discussed below. The second assumption introduces an uncertainty in the angular pattern of the produced radiation, but not in the overall shape of the spectrum (see e.g. Campitiello et al. 2017). The latter assumption is questionable in the case of near (or above) Eddington accretion, because the disc could become geometrically thicker close to the black hole.

Assuming a null spin implies an innermost radius of the circular orbit $\left(R_{\mathrm{ISCO}}=6 R_{\mathrm{g}}\right.$, where $R_{\mathrm{g}}$ is the gravitational radius) and a corresponding accretion efficiency (defined by $L=\eta \dot{M} c^{2}$ ) equal to 0.057 or 0.08 depending on whether relativistic effects are included or not. By increasing the spin, $R_{\mathrm{ISCO}}$ decreases, to become $R_{\mathrm{g}}$ when the dimensionless spin $a \sim 1$. Correspondingly, $\eta$ increases, reaching a theoretical maximum of 0.42 , which is however reduced to $\eta=$ 0.32 (Thorne 1974) when properly including the effects of accretion (and of photons produced by the disc falling into the black hole). The black hole spin has a negligible effect on the outer regions of the disc emitting in the infrared-optical band, but changes the emitting properties of the inner radii. In other words, for a given accretion rate and black mass, the disc around a rotating hole will produce the same amount of IR radiation but more UV than a Shakura-Sunyaev disc. 
The SED of J0100+2802 indeed shows a peak, allowing us to find the total luminosity associated with the accretion rate for the assumed efficiency $\eta$ and the black hole mass, since the peak frequency is associated with the temperature of the innermost orbits contributing to the observed spectrum. Applying the ShakuraSunyaev model (i.e. zero spin), we then find $M$ and $\dot{M}$. If we assume a non-zero and positive spin, the total luminosity can be produced with a reduced accretion rate ( $\eta$ is larger), but this implies that we underestimate the flux in the optical-IR bands (flux produced at larger radii). Therefore we have to increase the black hole mass (and therefore the surface of the disc) to make the disc 'colder' in order to fit the entire spectrum.

We consider the anisotropic emission of the disc, which follows a pattern $\propto \cos \theta$, and assume that object is observed under a viewing angle $30^{\circ}$ from the disc's normal (i.e. the average angle between $0^{\circ}$ and an assumed aperture angle of the torus of $45^{\circ}$ ). Along with the disc emission, we assume blackbody emission at a temperature $T_{\text {torus }}$ to model the torus emission. For the corona X-ray component, as explained above, we assume a power law of photon index $\Gamma=$ 2.5 ending with an exponential cut $\left(h v_{\text {cut }}=300 \mathrm{keV}\right)$, emitting a fraction $L_{\mathrm{X}} / L_{\mathrm{disc}} \sim 1 / 3$ of the optical-UV luminosity, here $L_{\mathrm{X}}$ is the total X-ray luminosity from the peak frequency of the disc emission to $\sim 1 \mathrm{MeV}$. The infrared and X-ray fluxes are assumed to be emitted isotropically.

Fig. 4 shows the infrared to X-ray spectral energy distribution (SED) of J0100+2802, together with the fitting model. The disc optical-UV luminosity is $L_{\text {disc }} \sim 3.7 \times 10^{47} \mathrm{erg} \mathrm{s}^{-1}$, which corresponds to 32 per cent of the Eddington luminosity, for a black hole mass of $M_{\mathrm{BH}}=9 \times 10^{9} \mathrm{M}_{\odot}$, slightly smaller than the estimate of $\mathrm{Wu}$ et al. (2015), but still consistent. The total X-ray luminosity (i.e. from the peak frequency of the disc emission to $\sim 1 \mathrm{MeV}$ ) is

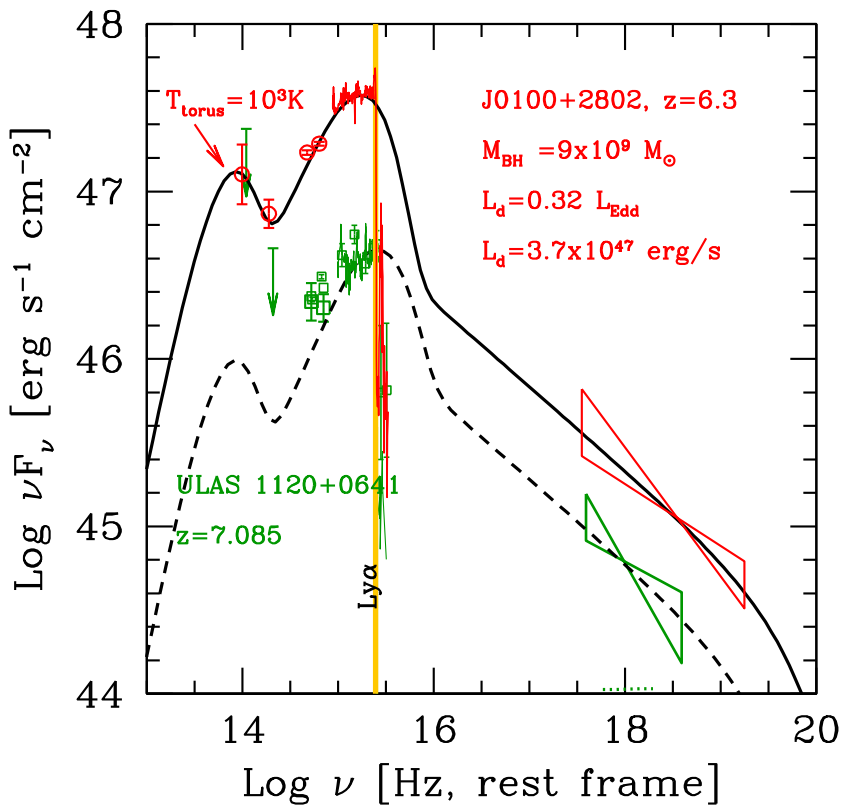

Figure 4. The spectral energy distributions of J0100+2802 (symbols in red) and our fitting model (solid black line) compared with the SED (symbols in green) and model (dashed black line) of ULAS J1120+0641. The vertical orange line labels the Ly $\alpha$ line. The inferred black hole mass and accretion luminosity for $\mathrm{J} 0100+2802$ are indicated. Infrared data are from WISE, optical spectra from the works by Mortlock et al. (2011) and Wu et al. (2015), respectively. X-rays of J0100+2802 are from this work. [A colour version of this figure is available in the online version.] $\sim 1 / 3$ of $L_{\mathrm{disc}}$. This gives $L_{\mathrm{X}}+L_{\mathrm{disc}} \sim 5 \times 10^{47} \mathrm{erg} \mathrm{s}^{-1}$, equivalent to $0.43 L_{\mathrm{Edd}}$. As explained above, the assumption of zero spin, implicit in the use of the Sakura-Sunyaev model, implies that the derived value of the black mass is a lower limit. This strongly suggests that the disc luminosity, including the rather large X-ray component, is sub-Eddington. The uncertainty of the derived black hole mass is $\sim 0.4$ dex, as shown in Fig. 5. In the figure we show the SED modelling of J0100+2802 corresponding to the same luminosity but with different masses.

\subsection{Comparison with ULAS J1120+0641}

Fig. 4 includes the SED of ULAS 1120+0641, the quasar with the largest measured redshift ( $z=7.085$ : Mortlock et al. 2011). In the far-infrared band we have only upper limits to the flux, which are not very constraining. Note also some discrepancy between the photometric and spectral data at the same frequencies. For the fit, we have given priority to the spectroscopic data.

This source is less luminous than $\mathrm{J} 0100+2802$ and its mass is smaller, according to the estimate obtained by fitting the SED. With the same accretion disc model as before, in fact, we obtain $M_{\mathrm{BH}}=$ $1.3 \times 10^{9} \mathrm{M}_{\odot}, L_{\mathrm{disc}}=3.9 \times 10^{46} \mathrm{erg} \mathrm{s}^{-1}=0.23 L_{\mathrm{Edd}}, L_{\mathrm{X}}=0.8 L_{\mathrm{disc}}$ $\sim 3 \times 10^{46} \mathrm{erg} \mathrm{s}^{-1}$ and $L_{\mathrm{X}}+L_{\mathrm{disc}}=0.41 L_{\mathrm{Edd}}$. As previously explained for $\mathrm{J} 0100+2802$, the value of the black hole mass should be taken as a lower limit. We conclude that both sources, despite the difference in black hole mass, share similar Eddington ratios and similar partition between optical-UV and X-ray luminosities. We can compare our results on ULAS J1120+0641 with those of Mortlock et al. (2011), who found $M_{\mathrm{BH}} \sim 2 \times 10^{9} \mathrm{M}_{\odot}$ (through the virial method) and a disc luminosity of $2.5 \times 10^{47} \mathrm{erg} \mathrm{s}^{-1}$ (applying a a fiducial bolometric correction taken from Willott et al. 2010). Differently from us, the results of Mortlock et al. (2011) indicate a slightly super-Eddington luminosity.

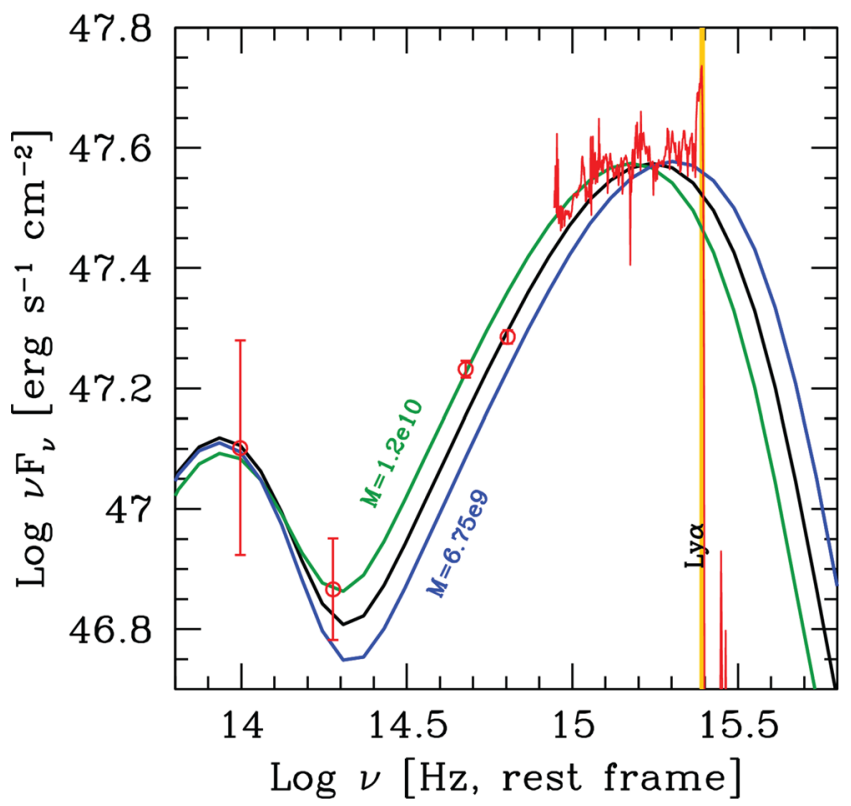

Figure 5. SED modelling of J0100+2802, corresponding to the same total luminosity but with three different masses (the solid black line is the one with mass $9 \times 10^{9} \mathrm{M}_{\odot}$ ). [A colour version of this figure is available in the online version.] 


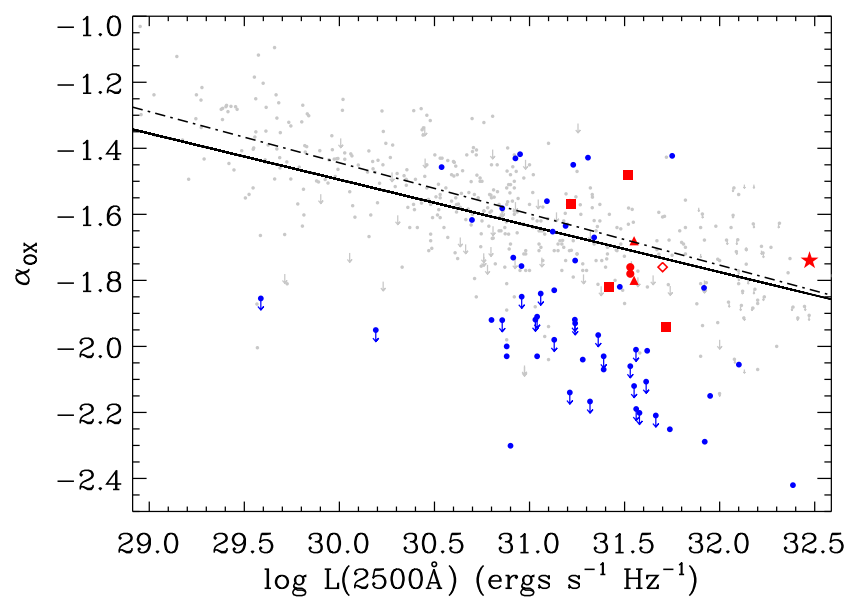

Figure 6. Location of J0100+2802 (red star) in the X-ray-to-optical powerlaw slope parameter $\alpha_{\text {ox }}$ versus 2500 - $\AA$ monochromatic luminosity. The grey dots are quasars from the samples of Just et al. (2007), Steffen et al. (2006) and Gibson, Brandt \& Schneider (2008). The blue dots are weak-line quasars and PHL 1811 analogues from Luo et al. (2015). The solid line represents the $\alpha_{\mathrm{ox}}-L_{2500} \AA$ relation from Just et al. (2007) and the dot-dashed line from Nanni et al. (2017). The red symbols represent high-redshift quasars with $z$ $>6.0$ from the literature (squares from Shemmer et al. (2006), filled circles for ULAS J1120+0641 (Moretti et al. 2014; Page et al. 2014), triangles for SDSS J1030 [Brandt et al. 2002; Farrah et al. 2004) and diamonds for SDSS J1148+5152 (Gallerani et al. 2017)]. [A colour version of this figure is available in the online version.]

\subsection{Comparison with other powerful quasars}

It is well-established that the X-ray-to-optical power-law slope parameter $\alpha_{\text {ox }}$ of quasars correlates significantly with the ultraviolet 2500- $\AA$ monochromatic luminosity $\left(L_{2500 \AA}\right.$ : Steffen et al. 2006; Just et al. 2007). For J0100+2802 with rest-frame 2500- $\AA$ flux density, $f_{2500} \AA$, estimated from Wu et al. (2015) and rest-frame $2-\mathrm{keV}$ flux density, $f_{2 \mathrm{keV}}$, estimated from the power-law model, we have parameter $\alpha_{\text {ox }}$ with value $-1.74 \pm 0.01$. In Fig. 6 , we show the location of $\mathrm{J} 0100+2802$ and the other highest-redshift quasars with $z>6$, for which we take $\alpha_{\text {ox }}$ and $L_{2500 \AA}$ from the literature (Brandt et al. 2002; Farrah et al. 2004; Shemmer et al. 2006; Moretti et al. 2014; Page et al. 2014; Gallerani et al. 2017), in the $\alpha_{\text {ox }}-L_{2500} \AA$ relation. It is clear that the SED of the ultraluminous J0100+2802 is not abnormal among the highest-redshift quasars and all quasars at $z>6$ follow the $\alpha_{\mathrm{ox}}-L_{2500 \AA}$ relation like low- and median-redshift quasars. As discussed in Nanni et al. (2017), which presents a systematic analysis of X-ray archival data of quasars at $z>5.5$, these results support the non-evolutionary scenario of SEDs of luminous quasars. For J0100+2802, the inferred value of $\alpha_{\mathrm{ox}}$ in Nanni et al. (2017) is $-1.88_{-0.02}^{+0.01}$, which is in agreement with ours considering the scatter of the $\alpha_{\mathrm{ox}}-L_{2500 \AA}$ relation.

With rest-frame equivalent width of $\operatorname{Ly} \alpha+\mathrm{Nv} \sim 10 \AA$ (Wu et al. 2015), J0100+2802 is one of the weak-line quasars (WLQs), which are a subclass of radio-quiet quasars that have almost extremely weak or undetectable emission lines (e.g. Fan et al. 1999; Meusinger \& Balafkan 2014, and references therein). A significant fraction ( $\sim 50$ per cent) of WLQs are distinctly X-ray weak compared with typical quasars (Shemmer et al. 2009; Wu et al. 2012; Luo et al. 2015). However, as shown in Fig. 6, J0100+2802 is not X-ray weak compared with the SEDs of other WLQs. J0100+2802, presented as an X-ray-normal weak-line quasar, provides constraints on the proposed hypotheses for the interpretation of weak-line quasars, such as a soft ionizing spectral energy distribution due to intrinsic
X-ray weakness or small-scale absorption (e.g. Leighly et al. 2007; Wu et al. 2012; Luo et al. 2015).

We did not detect variation of the X-ray emission for J0100+2802 in the $X M M-N e w t o n$ exposure and no signifiant variation was detected in the X-ray flux observed from XMM-Newton and Chandra observations. For this high-redshift radio-quiet luminous quasar, the non-detection of variation is not unexpected. Also, the results normally rule out the possibility of a jet-beaming effect in the observed X-ray brightness of J0100+2802, in which case there should be detected variations.

\section{SUMMARY}

With well-detected X-ray emission from an XMM-Newton observation, J0100+2802 presents as a peculiar high-redshift quasar in the X-ray with a relatively soft X-ray spectral shape. With the X-ray observation, the bolometric luminosity is calculated from spectral energy distribution modelling and the accretion rate is estimated to be sub-Eddington. The location in the $\alpha_{\mathrm{ox}}-L_{2500 \AA}$ relation indicates that it is an X-ray-normal quasar, in terms of either high-redshift quasars or weak-line quasars. The results from the XMM-Newton observation of J0100+2802 are meaningful for the study of quasar $\mathrm{X}$-ray properties, broad-band energy distribution and supermassive black hole formation and evolution at cosmic dawn.

\section{ACKNOWLEDGEMENTS}

Y-LA and LLF acknowledge support from the NSFC grants 11273060, 91230115 and 11333008 and State Key Development Program for Basic Research of China (No. 2013CB834900 and 2015CB857000). ACF and SAW acknowledge support from ERC Advanced Grant 340442. SAW was also supported by an appointment to the NASA Postdoctoral Program at the Goddard Space Flight Center, administered by USRA through a contract with NASA. XF acknowledges support from NSF grant AST 15-15115 and from the Institute of Astronomy, University of Cambridge through a Raymond and Beverly Sackler Distinguished Visitor program. FW and X-BW acknowledge support from NSFC grants 11373008 and 11533001 . We thank Tinggui Wang and Junxian Wang for helpful discussions. This work is based in part on observations obtained with $X M M-N e w t o n$, an ESA science mission with instruments and contributions directly funded by ESA Member States and NASA. We thank Dr Norbert Schartel for the allocation of $X M M$ observing time.

\section{REFERENCES}

Ai Y. L., Dou L. M., Fan X., Wang F. G., Wu X.-B., Bian F. Y., 2016, ApJL, 823,37

Ai Y. L., Dou L. M., Fan X., Wang F. G., Wu X.-B., Bian F. Y., 2017, ApJL, 841,32

Arnaud K. A., 1996, in Jacoby G. H., Barnes J., eds, ASP Conf. Ser. Vol. 101, Astronomical Data Analysis Software and Systems V. Astron. Soc. Pac., San Francisco, p. 17

Bartalucci I., Mazzotta P., Bourdin H., Vikhlinin A., 2014, A\&A, 566, 25

Brandt W. N. et al., 2002, ApJ, 569, 5

Calderone G., Ghisellini G., Colpi M., Dotti M., 2013, MNRAS, 431, 210

Campitiello S., Ghisellini G., Sbarrato T., Calderone G., 2017, MNRAS, preprint (arXiv:1702.00011)

Fabian A. C., Walker S. A., Celotti A., Ghisellini G., Mocz P., Blundell K. M., McMahon R. G., 2014, MNRAS, 442, 81

Fan X. et al., 1999, ApJ, 526, L57

Fan X., Carilli C. L., Keating B., 2006, ARA\&A, 44, 415 
Farrah D., Priddey R., Wilman R., Haehnelt M., McMahon R., 2004, ApJ, 611,13

Gallerani S. et al., 2017, MNRAS, 467, 3590

Gibson R. R., Brandt W. N., Schneider D. P., 2008, ApJ, 685, 773

Just D. W., Brandt W. N., Shemmer O., Steffen A. T., Schneider D. P., Chartas G., Garmire G. P., 2007, ApJ, 665, 1004

Kalberla P. M. W., Burton W. B., Hartmann D., Arnal E. M., Bajaja E., Morras R., Pöppel W. G. L., 2005, A\&A, 440, 775

Leighly K. M., Halpern J. P., Jenkins E. B., Casebeer D., 2007, ApJS, 173,

Luo B. et al., 2015, ApJ, 805, 122

Meusinger H., Balafkan N., 2014, A\&A, 568, A114

Moretti A. et al., 2014, A\&A, 563, 46

Mortlock D. J. et al., 2011, Nature, 474, 616

Nanni R., Vignali C., Gilli R., Moretti A., Brandt W. N., 2017, Astron. Astrophys., preprint (arXiv:1704.08693)

Page M. J., Simpson C., Mortlock D. J., Warren S. J., Hewett P. C., Venemans B. P., McMahon R. G., 2014, MNRAS, 440, 91

Planck Collaboration XVI, 2014, A\&A, 571, A16
Shakura N. I., Sunyaev R. A., 1973, A\&A, 24, 337

Shankar F., Weinberg D. H., Miralda-Escudé J., 2009, ApJ, 690, 20

Shemmer O. et al., 2006, ApJ, 644, 86

Shemmer O., Brandt W. N., Anderson S. F., Diamond-Stanic A. M., Fan X.,

Richards G. T., Schneider D. P., Strauss M. A., 2009, ApJ, 696, 580

Shen Y. et al., 2011, ApJS, 19445

Steffen A. T., Strateva I., Brandt W. N., Alexander D. M., Koekemoer A. M., Lehmer B. D., Schneider D. P., Vignali C., 2006, AJ, 131, 2826

Thorne K. S., 1974, ApJ, 191, 507

Volonteri M., 2010, A\&AR, 18, 279

Wang R. et al., 2017, ApJ, 835, 20

Willott C. J. et al., 2010, AJ, 140, 546

Wu J., Brandt W. N., Anderson S. F., Diamond-Stanic A. M., Hall P. B., Plotkin R. M., Schneider D. P., Shemmer O., 2012, ApJ, 747, 10

Wu X.-B. et al., 2015, Nature, 518, 512

This paper has been typeset from a $\mathrm{T}_{\mathrm{E}} \mathrm{X} / \mathrm{L} \mathrm{T}_{\mathrm{E}} \mathrm{X}$ file prepared by the author. 